\title{
A Discrete Electromechanical Model for Human Cardiac Tissue: Effects of Stretch-Activated Currents and Stretch Conditions on Restitution Properties and Spiral Wave Dynamics
}

\author{
Louis D. Weise ${ }^{1 *}$, Alexander V. Panfilov ${ }^{2}$ \\ 1 Department of Theoretical Biology, Utrecht University, Utrecht, The Netherlands, 2 Department of Physics and Astronomy, Ghent University, Ghent, Belgium
}

\begin{abstract}
We introduce an electromechanical model for human cardiac tissue which couples a biophysical model of cardiac excitation (Tusscher, Noble, Noble, Panfilov, 2006) and tension development (adjusted Niederer, Hunter, Smith, 2006 model) with a discrete elastic mass-lattice model. The equations for the excitation processes are solved with a finite difference approach, and the equations of the mass-lattice model are solved using Verlet integration. This allows the coupled problem to be solved with high numerical resolution. Passive mechanical properties of the mass-lattice model are described by a generalized Hooke's law for finite deformations (Seth material). Active mechanical contraction is initiated by changes of the intracellular calcium concentration, which is a variable of the electrical model. Mechanical deformation feeds back on the electrophysiology via stretch-activated ion channels whose conductivity is controlled by the local stretch of the medium. We apply the model to study how stretch-activated currents affect the action potential shape, restitution properties, and dynamics of spiral waves, under constant stretch, and dynamic stretch caused by active mechanical contraction. We find that stretch conditions substantially affect these properties via stretch-activated currents. In constantly stretched medium, we observe a substantial decrease in conduction velocity, and an increase of action potential duration; whereas, with dynamic stretch, action potential duration is increased only slightly, and the conduction velocity restitution curve becomes biphasic. Moreover, in constantly stretched medium, we find an increase of the core size and period of a spiral wave, but no change in rotation dynamics; in contrast, in the dynamically stretching medium, we observe spiral drift. Our results may be important to understand how altered stretch conditions affect the heart's functioning.
\end{abstract}

Citation: Weise LD, Panfilov AV (2013) A Discrete Electromechanical Model for Human Cardiac Tissue: Effects of Stretch-Activated Currents and Stretch Conditions on Restitution Properties and Spiral Wave Dynamics. PLoS ONE 8(3): e59317. doi:10.1371/journal.pone.0059317

Editor: Jordi Garcia-Ojalvo, Universitat Politecnica de Catalunya, Spain

Received November 22, 2012; Accepted February 13, 2013; Published March 19, 2013

Copyright: ( 2013 Weise, Panfilov. This is an open-access article distributed under the terms of the Creative Commons Attribution License, which permits unrestricted use, distribution, and reproduction in any medium, provided the original author and source are credited.

Funding: LDW is funded by the Netherlands Organization for Scientific Research (http://www.nwo.nl) (NWO Grant No. 613.000.604) of the research Council for Physical Sciences (EW). The funders had no role in study design, data collection and analysis, decision to publish, or preparation of the manuscript.

Competing Interests: The authors have declared that no competing interests exist.

* E-mail: L.D.Weise@uu.nl

\section{Introduction}

The heartbeat is governed by electrical waves of excitation that periodically propagate through the cardiac muscle and initiate its contraction. Abnormal electrical excitation of the heart may result in cardiac arrhythmias disturbing the heart's pumping function. Heart failure due to cardiac arrhythmias is a major cause of death in the industrialized world [1]. It is known that dangerous types of arrhythmias are caused by spiral waves of electrical excitation in the cardiac muscle [2-4].

Electrical waves of excitation are affected by the deformation of the heart via the mechano-electrical feedback phenomenon. It has been shown that the rapid stretching of cardiac tissue (mechanical stimulation) has a significant effect on the heart's functioning, for example, due to the initiation of electrical waves [5,6]. Important examples are "commotio cordis" [7,8], the phenomenon that an impact on the chest can cause arrhythmia; and the "precordial thump", the phenomenon that an impact on the chest of a patient may stop an arrhythmic heart condition [9]. Both phenomena are believed to be a result of an abrupt deformation of the heart, and the main effect of deformation on the electrical activity is considered to be transmitted via so-called stretch-activated ion channels. These channels produce depolarizing inward current as a response to stretch of the tissue [5]. The study of mechanoelectrical feedback is an important direction of research in current cardiac electrophysiology [10].

A valuable method to study mechano-electrical feedback is mathematical modeling allowing to study the coupled mechanical and electrical activity of the heart, which is a difficult problem in experimental research. Generic electromechanical models for heart tissue are successfully applied, for instance, to investigate the effect of mechano-electrical feedback on pacemaking and spiral wave activity [11-15] and to find mechanisms for the onset of spiral waves $[16,17]$. Yet, these generic models are limited to studies on a qualitative level, and more detailed models for cardiac tissue need to be developed. First steps in that direction have been made by coupling continuous mechanical models to biophysical models of cardiac excitation and contraction [18,19]. However, continuum mechanics is computationally demanding and makes it difficult to achieve high spatial and temporal resolution of the coupled electrical and mechanical processes. In this paper, we 
introduce an electromechanical model for human cardiac tissue which couples detailed biophysical models for cardiac excitation and contraction to a discrete mechanical model. We use an ionic model of excitation for human cardiac cells (Tusscher, Noble, Noble, Panfilov 2006 model) [20,21] and a biophysical model for excitation-contraction coupling (adjusted Niederer, Hunter, Smith, 2006 model) $[22,23]$. Our method applies a generic model for cardiac elasticity, an ideal crystal lattice of mass points connected with springs. The mass-lattice model describes a material which was introduced by Seth 1935 to discuss problems of finite strain [24]. The Seth material relation is an extension of the generalized Hooke's law to finite elasticity. To solve the mechanical equations we apply the Verlet integration [25] (explicit, finite difference backwards integration scheme), a method which is widely used in molecular dynamics simulations. The Verlet integration has first been used to solve mass-lattice models in cardiac elasticity by Mohr [26]. We applied this discrete mechanical description before in a model to study reactiondiffusion-mechanics systems [15], and use it here to set up an electromechanical model for cardiac tissue. An advantage of this method is its computational efficiency which allows to solve the coupled electromechanical equations with high spatiotemporal resolution.

To demonstrate the value of the discrete electromechanical model we apply it to study effects of deformation on basic properties of cardiac tissue, such as the action potential shape, restitution properties, and the dynamics of spiral waves. In this study we consider two mechanical conditions. The first one is a constantly stretched medium, a simple assumption which was widely used in initial studies on mechano-electric feedback [27,28], and mimics conditions such as dilated cardiomyopathies [27]. As a second condition, we assume a deforming medium which resembles deformation occurring as a result of cardiac contraction. We find that these stretch conditions have very different effects on the studied characteristics of excitation, and discuss the underlying mechanisms. The results of our application study may be important to understand how situations of increased mechanical load in the cardiac muscle alter the qualitative effect of stretchactivated currents.

\section{Methods}

\section{Model for Cardiac Excitation}

We use the 2006 version of the Tusscher Noble Noble Panfilov model for human epicardial myocytes (TP06) [20,21]. The model is given as the following reaction-diffusion equation for the transmembrane potential $V$

$$
\frac{\partial V}{\partial t}=D \Delta V-\frac{I_{\text {ion }}}{C_{m}}
$$

with the membrane capacitance density $C_{m}=2.0 \mu \mathrm{F} / \mathrm{cm}^{2}$, the diffusivity $D_{i j}=\Delta_{i j} \times 0.00154 \mathrm{~cm}^{2} / \mathrm{ms}$, and the transmembrane ion current

$$
\begin{aligned}
I_{i o n}= & I_{N a}+I_{K 1}+I_{t o}+I_{K r}+I_{K s}+I_{C a L}+I_{N a C a}+ \\
& I_{N a K}+I_{p C a}+I_{p K}+I_{b C a}+I_{b N a}+I_{s a c},
\end{aligned}
$$

where $I_{N a}$ is fast $\mathrm{Na}^{+}$current, $I_{C a L}$ is L-type $\mathrm{Ca}^{2+}$ current, and the $\mathrm{K}^{+}$currents are $I_{t o}$ (transient outward), $I_{K r}$ (rapid delayed rectifier), $I_{K s}$ (slow delayed rectifier), and $I_{K 1}$ (inward rectifier). Furthermore, $I_{N a K}$ is the $\mathrm{Na}^{+} / \mathrm{K}^{+}$pump current, $I_{N a C a}$ is the
$\mathrm{Na}^{+} / \mathrm{Ca}^{2+}$ exchanger current, $I_{p C a}, I_{p K}$ are plateau $\mathrm{Ca}^{2+}$ and $\mathrm{K}^{+}$currents, and $I_{b C a}, I_{b N a}$ are background $\mathrm{Ca}^{2+}$ and $\mathrm{Na}^{+}$ currents. The voltage dependency of ion channels is modeled [29] by gating variables with dynamics of the form

$$
\frac{d n}{d t}=\frac{n_{\infty}-n}{\tau}
$$

where $n_{\infty}$ describes the voltage-dependent steady state activation, and $\tau$ the voltage-dependent characteristic time for a respective gating variable. The TP06 model also describes $\mathrm{Ca}^{2+}$ dynamics of intracellular compartments of the sarcoplasmatic reticulum. A list of parameters and equations for these currents is given in [20].

In our model we add a stretch-activated depolarizing current $I_{s a c}$ which will be introduced in the section "Mechano-Electrical Feedback".

We will now describe the coupling of the electrical excitation process of the cardiomyocytes to their tension development.

\section{Model for Excitation-Contraction Coupling}

We model myocyte excitation-contraction coupling in our model with a numerically improved version of the Niederer, Hunter, Smith (NHS) model [22,23] adjusted to human cardiac tissue. The NHS model describes active tension in a sarcomere as a function of intracellular calcium concentration $\left[\mathrm{Ca}^{2+}\right]_{i}$, sarcomere length, and the rate of sarcomere length change, determinants which have been shown to substantially affect the active tension development (see [22] and references within).

The NHS model takes the dynamics of sarcomere length into account. We follow previous studies [12,15-17] and define as a pseudo normalized sarcomere length.

$$
\lambda:=\sqrt{\frac{A}{A_{0}}},
$$

where the $A$ is the surface area of a smallest area element in the model (see section "Mass-Lattice Model"), and $A_{0}$ is the surface area of such a smallest area element in undeformed state.

Adjustments for Human Ventricular Cells. We followed changes on the original version of the NHS model [22], which was originally set up using experimental data of rat and guinea pig hearts, that have been made in the work [18] to model human ventricular myocytes. These changes were explained in [18] by experimentally observed relaxation rates due to higher body temperatures [30]. The changes are a speeding up of myocyte relaxation rates to $\alpha_{r 1}=10 s^{-1}, \alpha_{r 2}=25 s^{-1}$, and adjusting the contractile tension by setting parameters $\mathrm{T}_{r e f}=125 \mathrm{kPa}$ (maximum contractile tension at resting length of sarcomere) and $\mathrm{pCa}_{50}=6.5\left(\mathrm{p}\left[\mathrm{Ca}^{2+}\right]_{i}\right.$ at half maximal contractile tension $)$.

Active Tension in Myocytes. The NHS model describes the tension $T_{a}$ development in cross bridges as.

$$
\begin{gathered}
T_{a}=\widehat{T} K\left(Q_{i}\right) \alpha, \\
\widehat{T}=T_{\text {ref }}\left(\frac{z}{z_{\max }}\right),
\end{gathered}
$$

where $\hat{T}$ is the length and velocity independent tension, $\left(z / z_{\text {max }}\right)$ is the fraction of available actin sites in a sarcomere $z$ to the maximal available actin sites $z_{\max }$ at a particular sarcomere length. Variable $z$ provides the coupling of the electrical and mechanical system 
and is found during integration of the NHS model where it is directly related to $\left[\mathrm{Ca}^{2+}\right]_{i}$, which is given by the electrical equations of the TP06 model. Scaling functions $\alpha$ and $K\left(Q_{i}\right)$ describe the sarcomere length and velocity dependencies of the total tension [22]. In particular, the function $\alpha$ models the influence of the dynamics of the cross bridge cycle and thin filaments in a sarcomere, and function $K\left(Q_{i}\right)$ accounts for the velocity dependency of tension development via a fading memory model.

It has been shown that in strongly coupled electromechanical models for cardiac tissue, in which the equations for excitation processes and mechanical processes are jointly solved, computational difficulties may occur due to the velocity and lengthdependency of a sarcomere [23]. To solve this problem, Niederer and Smith proposed the "update method" [23], where the functions $K\left(Q_{i}\right)$ and $\alpha$ are continuously calculated within the mechanical iteration algorithm to calculate the total tension $T_{a}$ via Eq.(5). In this paper, we apply the NHS model, adjusted for human cardiac tissue, together with the "update method". For a detailed model description and parameters, see $[22,23]$.

We will now describe the passive elastic properties of the medium.

\section{Mass-Lattice Model}

We use the mass-lattice framework introduced in [15]. The twodimensional lattice consists of material points connected by springs (Figure 1A). In this square lattice each mass point is connected to $N=8$ (if not at the boundary) direct neighboring mass points with springs that follow Hooke's law (Figure 1B). The equations of the model are.

$$
\begin{gathered}
\mathbf{F}_{12}=\frac{1}{\rho}\left(\frac{T_{a}(1)+T_{a}(2)}{2}\right) \frac{\mathbf{l}_{12}}{\left\|\mathbf{l}_{12}\right\|}, \\
\mathbf{f}_{1 a}=\left[c\left(\frac{\left\|\mathbf{l}_{12}\right\|-r_{0}}{r_{0}}\right)-d \frac{\left(\mathbf{i}_{12} \cdot \mathbf{l}_{12}\right)}{\left\|\mathbf{I}_{12}\right\|}\right] \frac{\mathbf{l}_{12}}{\left\|\mathbf{l}_{12}\right\|}+\mathbf{F}_{12}, \\
\mathbf{f}_{1 p}=\kappa\left[c\left(\frac{\left\|\mathbf{l}_{13}\right\|-\sqrt{2} r_{0}}{\sqrt{2} r_{0}}\right)-d \frac{\left(\mathbf{l}_{13} \cdot \mathbf{l}_{13}\right)}{\left\|\mathbf{l}_{13}\right\|}\right] \frac{\mathbf{l}_{13}}{\left\|\mathbf{l}_{13}\right\|},
\end{gathered}
$$

$$
\sum_{\alpha=1}^{N} \mathbf{f}_{i \alpha}=m \ddot{\mathbf{x}}_{i}=0
$$

Figure 1C illustrates main forces and the displacements of active and passive lattice springs connecting the mass point $\mathbf{1}$ to the mass points $\mathbf{2}$ and $\mathbf{3}$. Each mass point is connected to its 4 diagonal neighbors with "passive springs" (passive elastic properties), and to its 2 vertical and 2 horizontal neighbors with "active springs" (passive and active forces). Eq.(7) describes the excitationcontraction coupling of two neighboring mass points 1 and 2 connected with an active spring, where $T_{a}$ is active tension from Eq.(5), and $\rho$ is the mass point surface density (see section "Numerical Methods"). Eqs.(8),(9) describe forces $\mathbf{f}_{1 a}=-\mathbf{f}_{2 a}$ mediated through an active spring to mass points $\mathbf{1}$ and $\mathbf{2}$, and forces $\mathbf{f}_{1 p}=-\mathbf{f}_{3 p}$ mediated through a passive spring to mass points $\mathbf{1}$ and $\mathbf{3}$. In Eqs.(8),(9) the spring vectors are given by mass point's positions as $\mathbf{l}_{12}=\mathbf{x}_{2}-\mathbf{x}_{1}$ and $\mathbf{l}_{13}=\mathbf{x}_{3}-\mathbf{x}_{1}, r_{0}$ is the resting length of an active spring and $\sqrt{2} r_{0}$ the resting length of a passive spring, $\dot{\mathbf{i}}_{12}=\mathbf{v}_{2}-\mathbf{v}_{1}$ and $\dot{\mathbf{I}}_{13}=\mathbf{v}_{3}-\mathbf{v}_{1}$ are the time derivatives of the respective spring vectors $\mathbf{l}_{12}, \mathbf{l}_{13}$. Parameter $c$ is the stiffness constant, and $d$ is the damping parameter. Parameter $\kappa=1 / 2$ is the stiffness ratio between active and passive springs which causes the lattice to be macroscopically isotropic [31] for small deformations, and can be described by the generalized Hooke's law.

$$
\sigma_{i j}=C_{i j k l} \epsilon_{k l}=2 \mu \epsilon_{i j}+\lambda(\operatorname{tr\epsilon }) \delta_{i j}
$$

with the small strain tensor $\epsilon_{k l}$, Cauchy's stress tensor $\sigma_{i j}$, linear elasticity tensor $C_{i j k l}$, Kronecker delta $\Delta_{i j}$, and Lamé coefficients $\lambda$ and $\mu$. Krivtsov showed in [32] that the lattice can be approximated by the Seth material relation [24] for non-linear deformations which is given by Eq.(11) when the Almansi's finite strain tensor is used instead of the small strain tensor. Young's elastic modulus of cardiac tissue has been measured in an atomic force microscopy study $100 \pm 11 \mathrm{kPa}$ [33]. However, cardiac tissue provides a nonlinear elastic behavior for larger deformations, and we found that setting Young's elastic modulus to $125 \mathrm{kPa}$ in our model results in maximal deformations of springs of $15 \%$, similar to contracting cardiac cells. Thus we set the spring
A

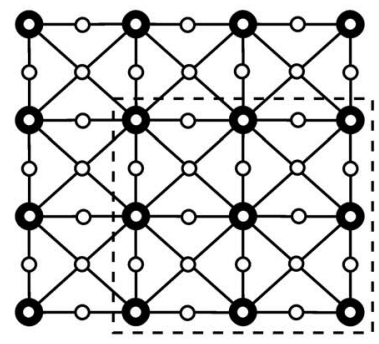

B

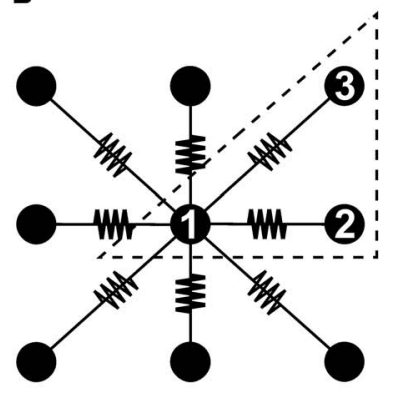

C

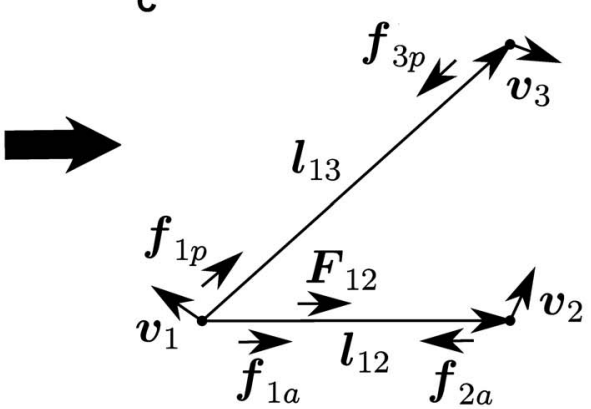

Figure 1. Coupled mechanical and electrical mesh. (A) Coupled mechanical and electrical mesh. The mass points are indicated as large black dots. The finite difference points to solve Eq.(1) are indicated as small white dots. The lattice springs are indicated as black lines. (B) Unit cell of the two-dimensional lattice. Mass point $\mathbf{1}$ and its four horizontal and vertical nearest neighbors and four diagonal next-nearest neighbors are connected with direct active and diagonal passive springs. Lattice springs are indicated by zigzagging lines (fat lines for active and thin lines for passive springs). Dotted contours indicate insets for the associated subfigures. (C) Vectors used in Eqs.(7)-(10) for calculating lattice interactions. Figure taken from [15].

doi:10.1371/journal.pone.0059317.g001 
stiffness $c$ accordingly to $125 \mathrm{kPa} / \rho$ (see section "Numerical Methods"). As we assume elastostatics $m$ and $d$ have no physical relevance and should be set to optimize computations. Following [15], we set $m$ to the identical numerical value of $c(m=0.125$ for $c=0.125 N$ compare section "Numerical Methods"), and set $d=30 \times c$ to achieve stable and efficient computations (see section "Model Validation").

In section "Model for Excitation-Contraction Coupling" we defined a pseudo normalized sarcomere length $\lambda$ in terms of the surface area of a smallest area element $A$ in the lattice (see Eq.(4)), which is a quadrilateral formed by 4 direct neighboring mass points connected with active springs (see Figure 1A). Parameter $A_{0}$ is the surface area of such a smallest area element in the undeformed model, $A_{0}=r_{0}^{2}$ (see Figure 1A).

We will now describe how we model mechano-electrical feedback via stretch-activated currents.

\section{Mechano-Electrical Feedback}

The deformation of a cardiomyocyte affects its excitation processes. It has been shown in studies of excised cardiac tissue and the whole heart that a direct electrophysiological influence of the deformation of cardiac tissue is a depolarizing stretch-activated current $I_{\text {sac }}$ (compare Eq.(2)) through stretch-activated ion channels [5]. Experimental studies have shown that these channels are activated instantaneously with mechanical stretch and follow a linear current-voltage relationship [34,35]. Linear, time-independent models have been proposed for $I_{s a c}[27,28]$, and have been used in other electromechanical models $[12,14,18]$. Following these previous studies we use.

$$
I_{s a c}=G_{s} \frac{(\lambda-1)}{\left(\lambda_{\max }-1\right)}\left(V-E_{S}\right), \text { for } \lambda>1(\text { stretch })
$$

where $G_{s}$ is the maximal conductance, and $E_{s}$ is the reversal potential of the stretch activated channels. For $E_{S}$ values around $[-20 ; 0] m V$ have been reported [36,37], and we set $E_{s}=0 m V$. It has been shown that $G_{S}$ is within 0 to $100 S / F[5,38]$, and in this paper we vary $G_{S}$ in this range to study the influence of $I_{S}$ on several properties. Parameter $\lambda_{\max }$ is the maximal pseudo normalized sarcomere length which we set to $\lambda_{\max }=1.1$ as in [18].

\section{Numerical Methods}

We solved the model applying an explicit Euler method for the TP06 and NHS models, and Verlet integration [25] for the mechanical model. After each Euler computation of the electrical system a new $\left[\mathrm{Ca}^{2+}\right]_{i}$ is obtained, and a length and velocity independent tension $\hat{T}$ is computed via Eq.(6). $\hat{T}$ is then passed to the mechanics model, where the mechanical equations are solved, using Verlet integration time step $m \tau=0.01$, until the sum of forces on each mass point is smaller than threshold $t h r=0.05 \mathrm{kPa} / \rho$. The Verlet computation of the position of a mass point $i$ for integration time $\tau+m \tau$ is

$$
\mathbf{x}_{i}(\tau+m \tau)=2 \mathbf{x}_{i}(\tau)-\mathbf{x}_{i}(\tau-m \tau)+\ddot{\mathbf{x}}_{i}(\tau) \times(m \tau)^{2},
$$

where $m \tau=0.01$ is the Verlet integration time step and $\tau$ is the integration time. For the very first time step, we use

$$
\mathbf{x}_{i}(0+m \tau)=\mathbf{x}_{i}(0)+\frac{1}{2} \ddot{\mathbf{x}}_{i}(0) \times(m \tau)^{2} .
$$

The acceleration of a mass point $\ddot{\mathbf{x}}_{i}(\tau)$ is given by Eq.10. At each time step the velocities of the mass points are calculated by

$$
\mathbf{v}_{i}(\tau)=\frac{\mathbf{x}_{i}(\tau)-\mathbf{x}_{i}(\tau-m \tau)}{m \tau} .
$$

During the mechanical iteration algorithm the length and velocity dependent tension scaling functions $K\left(Q_{i}\right)$ and $\alpha$ of the total tension $T_{a}$ which is computed via Eq.(5) are updated together with the mesh configuration using the "update-method" [23]. We found that numerical difficulties can occur in situations when the damping force in a spring exceeds the Hooke's force, for example in an active spring (see section "Mass-Lattice Model").

$$
d \frac{\left(\mathbf{i}_{12} \cdot \mathbf{l}_{12}\right)}{\left\|\mathbf{l}_{12}\right\|}>c\left(\frac{\left\|\mathbf{l}_{12}\right\|-r_{0}}{r_{0}}\right),
$$

which in turn causes slow convergence of the iterative algorithm requiring in some cases thousands of iterations before convergence. However, we found that good convergence can be achieved by setting the absolute damping force to $10 \%$ of the Hooke's spring force (for springs for which condition of Eq.(13) is true). With this, we observe a significant improvement - typically the mechanical system converges within 10-20 iterations. In this paper the "update method" is applied within the Verlet routine on discrete nodes, whereas in the original work [23] the method is used within the Newton algorithm to solve equations of continuum mechanics. Moreover, here the actual sarcomere length was not used for the mechano-electrical feedback calculation, but a pseudo normalized sarcomere length $\lambda$ from Eq.(4). For simulations we used an Euler integration space step from $\Delta x=\Delta y=0.025 \mathrm{~cm}$ to $0.05 \mathrm{~cm}$ and Euler integration time step of $\Delta t=0.02 \mathrm{~ms}$. We computed a quadratic grid of up to $403 \times 403$ finite difference points (and up to $201 \times 201$ mass points) using no-flux boundary conditions modeling a thin quadratic layer of $10 \times 10 \mathrm{~cm}^{2}$ cardiac tissue. The spring stiffness $c$ and mechanical threshold $t h r$ are functions of the mass point density. Mass point density $\rho$ is function of the coarseness of the mass-lattice model, here we set the resting length of an active spring to be as long as two finite difference integration steps $r_{0}=2 \times \Delta x$ (see Figure 1A), thus the mass point density is

$$
\rho=\frac{1}{r_{0}^{2}}=\frac{1}{4 \times \Delta x^{2}},
$$

where the factor $1 / 4$ is the ratio of mesh coarseness (\#mechanical points/\#electrical points) in the model. For an Euler space step of $\Delta x=0.05 \mathrm{~cm}$, the mass point density is $\rho=100 \mathrm{~cm}^{-2}$, spring stiffness is $c=0.125 \mathrm{~N}$, and the mechanical threshold is $t h r=0.05 \mathrm{mN}$. The boundaries of the medium were fixed in space modeling isometric contraction to mimic isovolumic phases in the cardiac cycle, a common assumption which was used in similar electromechanical studies [12,14-16].

\section{Model Validation}

The numerical coupling and integration of the Euler and the Verlet scheme require the choice of several parameters. We will first discuss the integration parameters and then parameters for the coupling of the numerical grids to assure efficient and stable computations. 
Integration Parameters. It has been shown that the TP06 model together with the improved NHS model coupled with a whole heart continuum mechanics model can be stably integrated with the Euler method using integration parameters $\Delta t=0.02 \mathrm{~ms}$ and $\Delta x=0.05 \mathrm{~cm}$ [18]. We use Euler integration steps of $\Delta t=0.02 \mathrm{~ms}$ and $\Delta x=0.05 \mathrm{~cm}$ as in [15] for most computations, and use a stricter setting $\Delta x=0.025 \mathrm{~cm}$ for simulations on spiral wave dynamics. To validate the usage of larger space step $\Delta x=0.05 \mathrm{~cm}$ we performed simulations of our main results on potential shape, and restitution properties using $\Delta x=0.025 \mathrm{~cm}$. In these simulations we found that our setting of Euler integration parameters yields consistent results. We update the mechanical configuration after each Euler step, and achieve stable and accurate integration of the coupled electromechanical model. For solving the mechanical Eqs.(8)-(10) we use a Verlet integration time step of $m \tau=0.01$ (as in [15]). We find that this setting allows efficient and stable computations of new configurations of the mechanical grid for this paper's simulations.

Damping-Stiffness-Ratio. The system of coupled, damped, driven, mechanical oscillators described by Eqs.(8)-(10) has been shown in [15] to allow fast stable convergence of the lattice mass points to their new configuration in a similar application as in this paper. In this work we found that setting the damping-stiffness ratio $d / c=30$ (dimensionless) as in [15] yields stable and efficient computations of mechanical mesh configurations in most situations. However, we found that in some situations (e.g. under external stimulation) we get numerical difficulties when Eq.(13) is true even for smaller values of the damping-stiffness ratio $d / c$ (e.g. $d / c=1)$. Thus, we apply $d / c=30$ together with the stability criterion outlined in the section "Numerical Methods" for all this paper's simulations.

Electrical and Mechanical Grids. In [15] we applied a method to validate the mesh coupling of the finite difference mesh and the mechanical mesh via an error norm defined by residues of mass point trajectories. This method allowed us to find accurate coupling parameters. We found in these validation experiments that the usage of a coarser finite difference mesh compared to the mechanical mesh as shown in Figure 1A allows accurate computations. This is, because changes in tension and strain are typically distributed more smoothly in space than electrical variables. For example, the upstroke of an electrical excitation wave has a length of the order of one to two millimeters, whereas the mechanical tension changes over a range of few centimeters. In this paper, we performed simulations to test how a change in resolution of the mechanical mesh compared to the electrical system affects the main results of the paper, and we found that our parameter setting yields consistent results. We also found in [15] that our mechanics model converges better with a frequent update rate, and therefore we also choose here to update the mechanical mesh after each time the electrical system was solved (every $\Delta t=0.02 \mathrm{~ms}$ ). We performed a convergence study to determine a suitable value for the threshold of convergence for the mechanical problem for numerical step sizes. For this we halved and quartered the value for $t h r$, and found qualitatively same results (influence of stretch on action potential, restitution properties, spiral wave dynamics). Thus we set $t h r=0.05 \mathrm{kPa} / \rho$, e.g. for $\Delta x=0.05 \mathrm{~cm}$, $t h r=0.05 \mathrm{mN}$, and for $\Delta x=0.025 \mathrm{~cm}, \mathrm{thr}=0.0125 \mathrm{mN}$.

Mesh Initial Conditions. The main determinant of cardiac contraction is the $\left[\mathrm{Ca}^{2+}\right]_{i}$ transient, and it is necessary to set initial conditions of the TP06 model so that it describes a steady state $\mathrm{Ca}^{2+}$ dynamics of a working cardiac cell. We found that one should carefully approach this problem as establishing of such a steady state can take a substantial period of time. To demonstrate this, we performed a numerical experiment on a single, non- deforming cell in which we stimulated it with a frequency of $2 \mathrm{~Hz}$ by setting $V=0 \mathrm{mV}$ for one time step $0.02 \mathrm{~ms}$. We see in Figure $2 \mathrm{~A}$ that it requires a long time to reach steady state dynamics for $\left[\mathrm{Ca}^{2+}\right]_{i}$. Figure 2B illustrates the $\left[\mathrm{Ca}^{2+}\right]_{i}$ transient after $30 \mathrm{~s}$ of applying the stimulation protocol. According to this simulation we adjusted initial conditions of the TP06 model: $\left[\mathrm{Ca}^{2+}\right]_{i}=0.11 \mu M ;[\mathrm{CaSR}]=3.77 m M$; CaSS $=0.2 \mu M$. Note that these calcium concentrations were taken at peak values of $\left[\mathrm{Ca}^{2+}\right]_{i}$, and that in following numerical experiments we performed additional initialization procedures.

Figure 3 shows an electromechanical pulse of a single fiber during isometric contraction. One can see that as in experimental records [39] the tension is slightly delayed from the $\left[\mathrm{Ca}^{2+}\right]_{i}$ transient, the fiber produces a maximal contractile tension of $\approx 85 \mathrm{kPa}$ approximately after $100 \mathrm{~ms}$ after the upstroke of the action potential.

\section{Results}

We applied our discrete electromechanical model to study the effects of stretch-activated currents and stretch conditions on action potential duration (APD) and conduction velocity (CV) restitution, and spiral wave dynamics. The results of these studies are shown in this section.

We consider two mechanical conditions, a constantly stretched medium, and a contracting medium. The condition of sustained stretch in the medium has been assumed previously, for example, in a model to study how dilated cardiomyopathies may affect defibrillation efficacy [27], and in a model to study the effect of mechano-electrical feedback on the action potential of ventricular cells [28]. However, under normal physiological conditions cells are not constantly stretched, but contract during most of the action potential. It is interesting to note, that as an experimental condition, constantly applied mechanical load is often applied to study effects of mechano-electrical feedback, for example, in cardiac cell cultures [40] or animal models [41]. Therefore, we perform studies both, in a constantly stretched medium, and in a contracting medium to investigate the effects of different mechanical conditions.

\section{Action Potential Shape and Restitution Properties}

Constant stretch. We used a $10 \mathrm{~cm} \times 10 \mathrm{~cm}$ medium which we assumed stretched to $\lambda_{\max }$. From Eq.(12) we see that in this situation every cell in the medium experiences $I_{s a c}=G_{s}\left(V-E_{s}\right)$, thus we apply $I_{s a c}$ to every cell without actually deforming the medium. We initiated a train of traveling plain waves with a period of $1 s$ to study the influence of $I_{s a c}$ on characteristics of the action potential for $G_{s}$ from 0 to $25 S / F$. In Figure 4 A we show the shape of the action potential of the cell in the center of the medium for different $G_{s}$ after $50 s$ application of the stimulation protocol. From Figure $4 \mathrm{~A}$ we see that increasing $G_{s}$ causes an increase in the resting potential in the medium and increase in APD. In particular, Figure 4B shows that increase of $G_{s}$ from 0 to $25 \mathrm{~S} / \mathrm{F}$ increases the resting potential by $20 \mathrm{mV}$ (from $-86 \mathrm{mV}$ to $-66 \mathrm{mV}$ ). Figure $4 \mathrm{C}$ shows that increasing $G_{s}$ from 0 to $25 \mathrm{~S} / \mathrm{F}$ increases APD from $311 \mathrm{~ms}$ to $361 \mathrm{~ms}$. This effect occurs as a larger $G_{s}$ causes a stronger depolarizing $I_{s a c}$. As a result of stronger $I_{s a c}$ the resting potential increases, and during an action potential $I_{s a c}$ counteracts repolarizing currents elongating the APD.

The effect of $I_{s a c}$ on the upstroke of the action potential in the constantly stretched medium is illustrated in Figure 5. We see in Figure $5 \mathrm{~A}$ that the upstroke peak and slope decreases with increasing $G_{S}$, and that for $G_{S}>15 S / F$ no typical sodium driven 

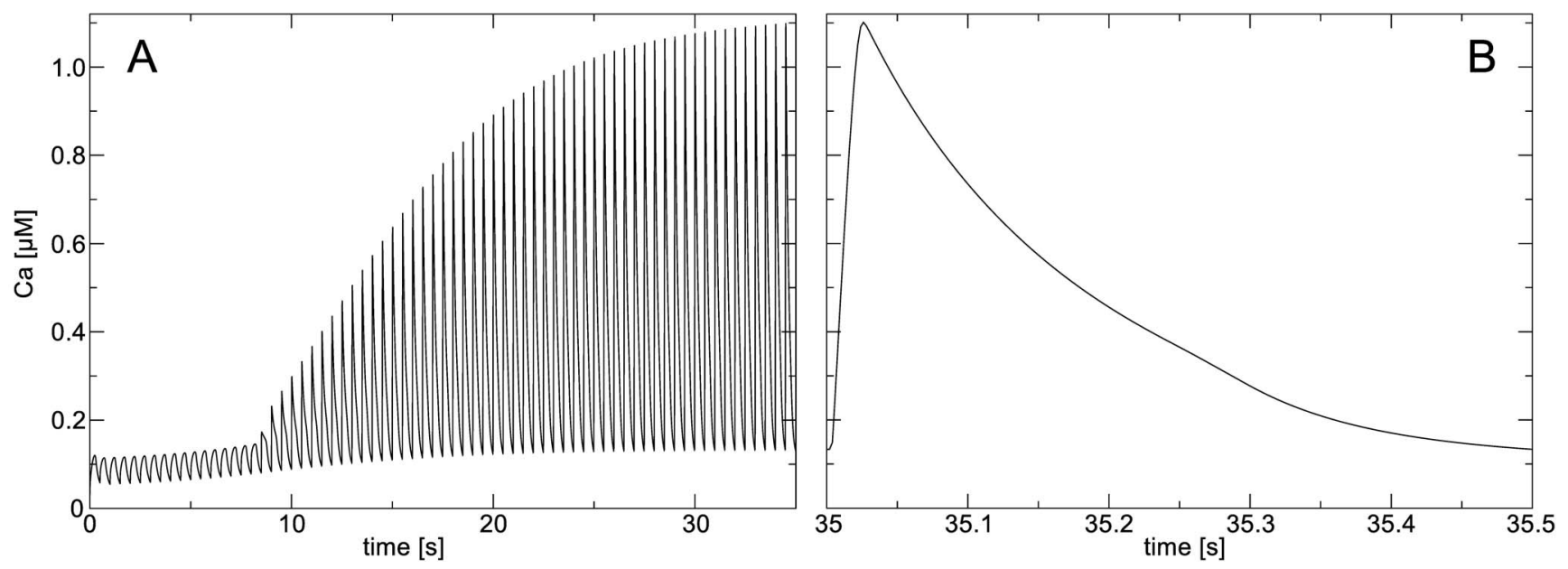

Figure 2. Mesh initialization (A) $\left[\mathrm{Ca}^{2+}{ }_{i}\right]$ transient during pacing experiment. $\left[\mathrm{Ca}^{2+}\right]_{i}$ is shown for a single non-deforming cell undergoing $2 \mathrm{~Hz}$ pacing. (B) Steady state $\left[\mathrm{Ca}^{2+}{ }_{i}\right]$ transient. $\left[\mathrm{Ca}^{2+}\right]_{i}$ is shown for a single non-deforming cell after $35 \mathrm{~s}$ of $2 \mathrm{~Hz}$ pacing. doi:10.1371/journal.pone.0059317.g002

upstroke takes place. In Figure 5B the upstroke slope is shown as a function of $G_{s}$. We see that for $G_{S}>20 S / F$ the upstroke slope drops to values under $50 \mathrm{~S} / F$ which is much lower than the sodium driven upstroke in the TP06 model without deformation. This effect of $G_{s}$ on the upstroke can be explained by a depletion of fast sodium channels via the accommodation phenomenon, a decrease of opening probability of fast sodium channels due to sub-threshold depolarization [29]. In a previous study [28], which also assumed a constantly stretched medium, similar effects of $I_{s a c}$ on the APD, upstroke and resting potential were found. Moreover, it was shown previously in Langendorff-perfused rabbit hearts that sustained volume load causes increased resting potential and decreased the slope of the action potential upstroke [41].

To measure restitution properties in the constantly stretched medium we applied the same setup as above, but varied the stimulation period from $0.33 s$ to $3 s$. We measured CV from the difference in front arrival times between two points, one at the center, and the other $1.5 \mathrm{~cm}$ further in propagation direction. Figure 6 illustrates the APD and CV restitution. We see from

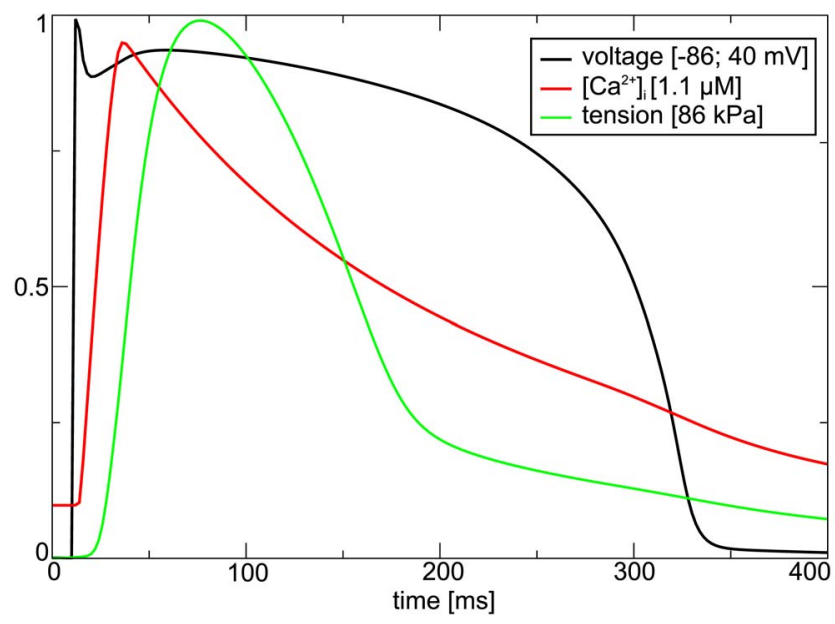

Figure 3. Electromechanical activity of an isolated fiber. A pulse is initialized at time $10 \mathrm{~ms}$ by setting voltage to $0 \mathrm{mV}$ for $0.02 \mathrm{~ms}$. Fiber was kept at its resting length during the simulation. doi:10.1371/journal.pone.0059317.g003
Figure 6A that increasing $G_{s}$ increases the APD. For $G_{s}$ between 0 and $10 S / F$ the slope of the APD restitution curve is not affected much, and APD grows continuously for longer stimulation periods to a plateau. For $G_{S}$ between 15 and $25 S / F$ and a stimulation period longer than $1 s$ we see that an increasing, slightly negative slope of the APD restitution curve is caused. From Figure 6B we see, that increasing $G_{s}$ causes decreasing CV. For stimulation intervals shorter than $0.75 s$ we observe that increasing $G_{s}$ causes a higher steepness of the $\mathrm{CV}$ restitution curve. Restitution curves for $G_{s}$ between 0 and $20 S / F$ are monotonically increasing for longer stimulation intervals; yet, for $G_{S}=25 S / F$ we see a biphasic shape with a local maximum of around $0.75 s$, and slightly negative slope of the $\mathrm{CV}$ restitution curve for longer stimulation interval. We also see that the minimal period of excitation increases with increase of $G_{s}$ from about $0.33 s$ for $G s \leq 10 S / F$ to about $0.5 s$ for $G_{s}>10 S / F$. In a previous study [27], where also constant stretch was assumed in the medium, increasing $G_{s}$ also caused longer APD and decreased CV.

Contracting Medium. For the simulations in a contracting medium we applied the same system size, stimulation protocol, and parameter setting as for the constantly stretched medium; however, the medium is deforming due to excitation-contraction waves, and the boundaries are fixed space (see section "Numerical Methods"). In Figure 7A we show how the shape of the action potential is affected by $I_{s a c}$. We see that increasing $G_{s}$ in from 0 to $100 S / F$ causes no substantial increase in the resting potential and in APD. Another effect of increasing $G_{s}$ is a linear increase in the transmembrane potential starting $\approx 50 \mathrm{~ms}$ prior the upstroke. For example, for $G_{S}=100 S / F$ the transmembrane potential increases to $-77 \mathrm{mV}$, which is well under the threshold of excitation, that is at $\approx-60 \mathrm{mV}$. In Figure 7B we illustrate the effect of $I_{s a c}$ on APD. One can see that increasing $G_{s}$ from 0 to $100 S / F$ increases APD by $0.45 \mathrm{~ms}$. This effect of $I_{s a c}$ on APD is small in the studied parameter range compared to the constantly stretched medium, where increasing $G_{s}$ from 0 to $25 S / F$ resulted in increase of APD by $50 \mathrm{~ms}$.

In Figure $7 \mathrm{C}$ we illustrate the effect of $I_{\text {sac }}$ on the action potential upstroke in the deforming medium. We can see that the upstroke peak decreases for increasing $G_{s}$; from $28.3 \mathrm{mV}$ (for $G_{s}=0 S / F$ ) to $22.1 \mathrm{mV}$ (for $G_{s}=100 S / F$ ). This decrease in upstroke peak is small compared to the constantly stretched medium, where an increase of $G_{S}$ from $0 S / F$ to $10 S / F$ caused a 

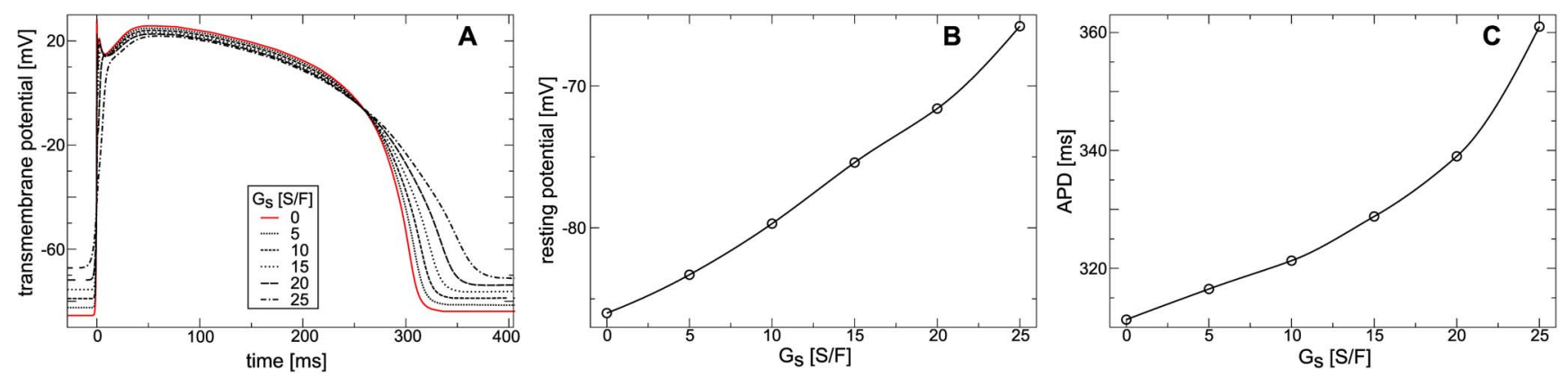

Figure 4. Effect of stretch-activated currents on the action potential in constantly stretched medium. (A) Action potential vs $G_{s}$. (B) Resting membrane potential vs $G_{s}$. (C) APD vs $G_{s}$. Traveling plain waves were periodically $(1 \mathrm{~Hz})$ induced in a medium held at $\left(\lambda_{\max }\right)$ for different $G_{s}$. Action potentials were measured after $50 \mathrm{~s}$. Resting potential was measured in the medium without external stimulations. APD was measured at $90 \%$ recovery.

doi:10.1371/journal.pone.0059317.g004

decrease of the upstroke amplitude by $15.0 \mathrm{mV}$ (compare Figure 5A). Furthermore, in Figure 7D we illustrate the maximal upstroke slope against $G_{s}$. The maximal upstroke slope decreases for increasing $G_{s}$. The effect of $I_{s a c}$ is small compared to the constantly stretched medium. In particular, increasing $G_{s}$ from 0 to $25 S / F$ in the contracting medium decreases the upstroke slope from $326.6 \mathrm{~V} / \mathrm{s}$ to $302 \mathrm{~V} / \mathrm{s}$, whereas in the constantly stretched medium an increase of $G_{S}$ from $0 S / F$ to $5 S / F$ causes a decrease to $269 \mathrm{~V} / \mathrm{s}$.

Figure 8A illustrates the effect of $I_{s a c}$ on the APD restitution in the contracting medium. From Figure $8 \mathrm{~A}$ we see a small effect of $I_{s a c}$ on the APD. Only for a stimulation period shorter than $1 s$ we see a small decrease in steepness of the restitution curve with increasing $G_{s}$.

Figure 8B illustrates the effect of $I_{s a c}$ on $\mathrm{CV}$ restitution in the contracting medium. We see, that for a stimulation period longer than $2 s$ stretch activated currents have only little effect on GV. This is because for a slow stimulation period the effects of deformation caused by a preceding wave progressively decrease. For stimulation periods shorter than $0.5 s$, a steep positive $\mathrm{CV}$ restitution is present for all measured values of $G_{s}$, and the slope of the $\mathrm{CV}$ curve increases when $G_{s}$ is larger. For stimulation periods between $1 s$ and $2 s$ we see that contraction results in negative $\mathrm{CV}$ restitution slopes: a higher periodic stimulation causes higher wave velocities. Note that $\mathrm{CV}$ depends on the position it is measured, as the medium before the wave is depolarized by $I_{s a c}$. Here we used an average $\mathrm{CV}$ to illustrate the abnormal CV restitution.

Effect of Mechanical Conditions. Let us now compare the results for a constantly stretched and contracting medium. We found, that under both, dynamical and static stretch conditions, increasing $G_{s}$ causes an elongation of the APD; however, in the deforming medium the effect is much smaller than in the constantly stretched medium (compare Figures $6 \mathrm{~A}$, and 4 with Figures $7 \mathrm{~B}$, and $8 \mathrm{~A}$ ). Furthermore, the results of the $\mathrm{CV}$ restitution in the constantly stretched and the contracting medium are significantly different. We can explain these differences by substantially different time courses of stretch in a constantly stretched and in contracting tissue. Figure 9 illustrates the shape of action potential, stretch activated current, and deformation of the medium for a single cell which is subject to a constant stretch (similar to Figure 4), and for a cell in a contracting medium, in which we measured the APD restitution shown in Figure 8. In both setups the cell was paced at $1 \mathrm{~Hz}$. We see that $I_{\text {sac }}$ in a constantly stretched cell is active during the entire action potential, and $I_{\text {sac }}$ has a substantial negative value (inward current) at the waveback, which results in APD prolongation. On the contrary, for a cell in the contracting medium $I_{s a c}$ is absent at the waveback. This is because at this phase of the action potential the cell is
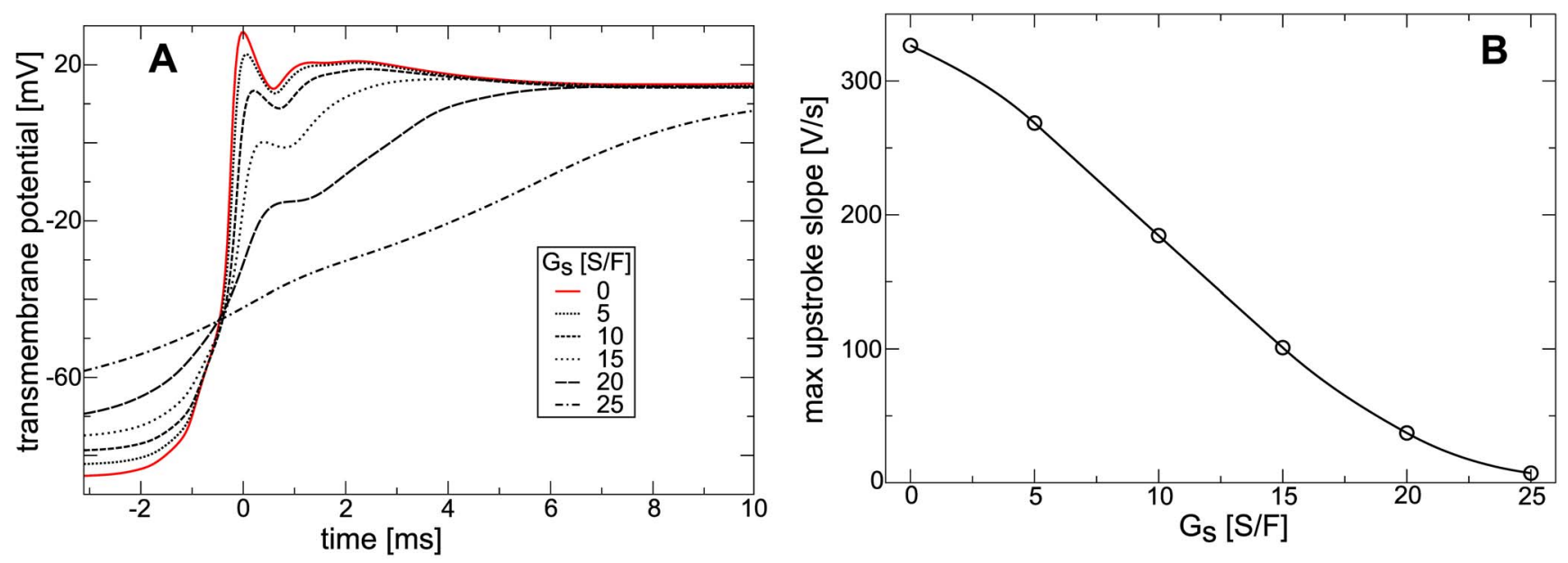

Figure 5. Effect of constant stretch on the action potential upstroke. (A) Upstroke of action potential vs $G_{s}$. (B) Maximal upstroke slope vs $G_{s}$. Protocol was as in Figureô 4.

doi:10.1371/journal.pone.0059317.g005 

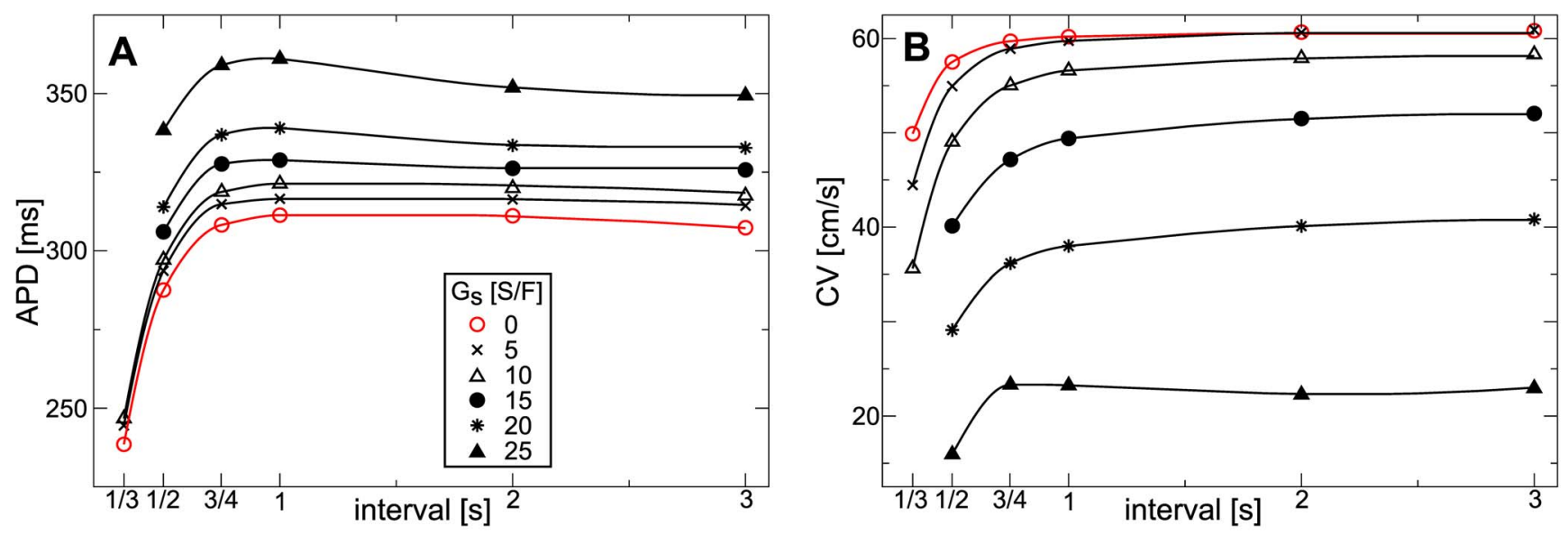

Figure 6. Dependence of restitution on stretch-activated currents in constantly stretched medium. (A) APD restitution vs $G_{s}$. (B) CV restitution vs $G_{s}$. Same parameters were used as in Figureô 4. doi:10.1371/journal.pone.0059317.g006

contracting, and thus no stretch activated current is produced. As a result of that difference, the APD for a cell in the deforming tissue is only slightly longer than that of a cell in a non-deforming tissue. Some elongation of the APD at increased $G_{s}$ can be explained with the negative current prior/during the upstroke of the action potential which slightly decreases the sodium current via
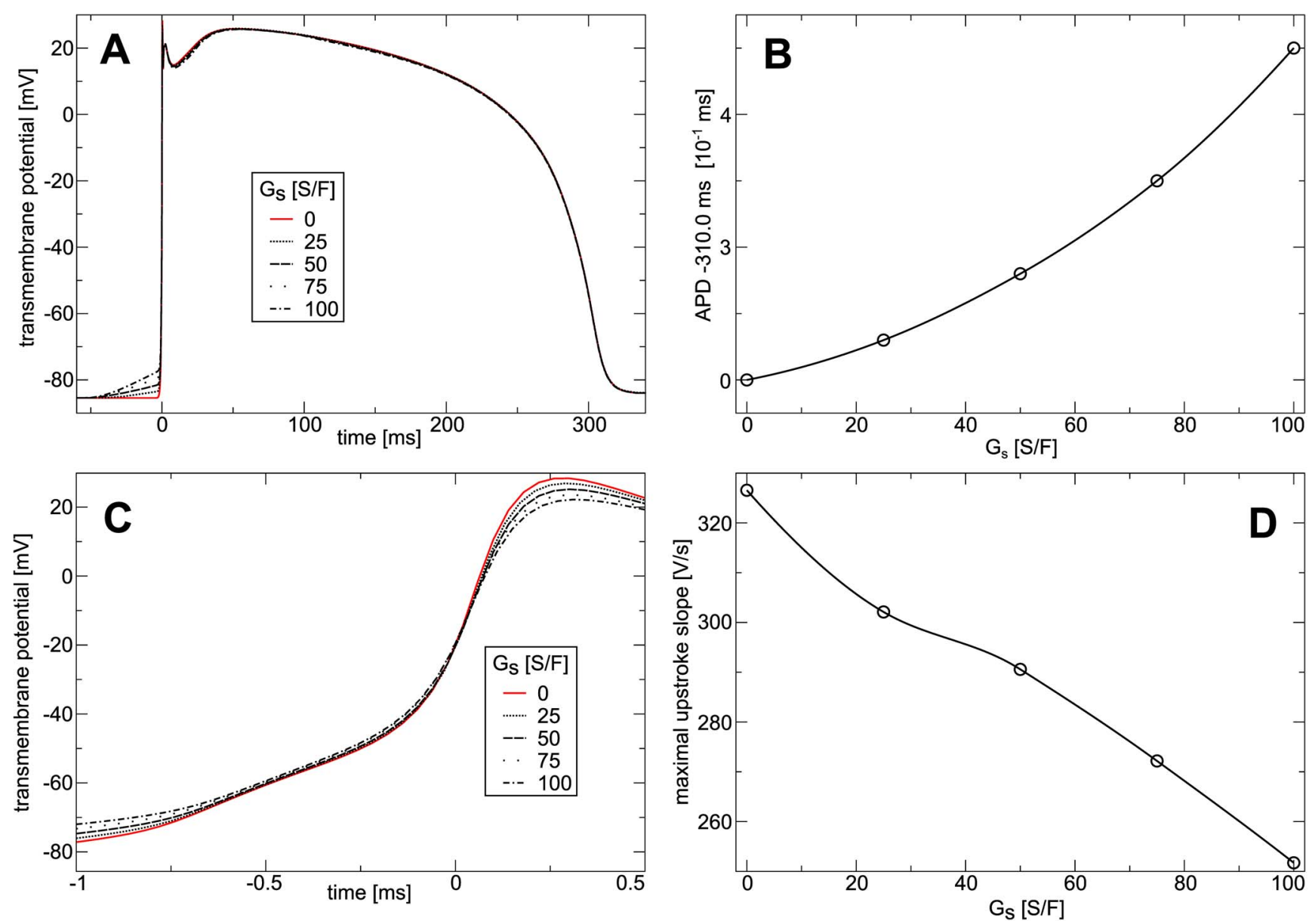

Figure 7. Effect of stretch-activated currents on the action potential in contracting medium. (A) Action potential vs $G_{s}$. (B) APD vs $G_{s}$. (C) Upstroke of action potential vs $G_{s}$. (D) Maximal upstroke slope vs $G_{s}$. Traveling plain waves were periodically $(1 \mathrm{~Hz})$ induced for different $G_{s}$. Action potentials were measured after $50 \mathrm{~s}$. Resting potential was measured in the medium without external stimulations. APD was measured at $90 \%$ recovery.

doi:10.1371/journal.pone.0059317.g007 

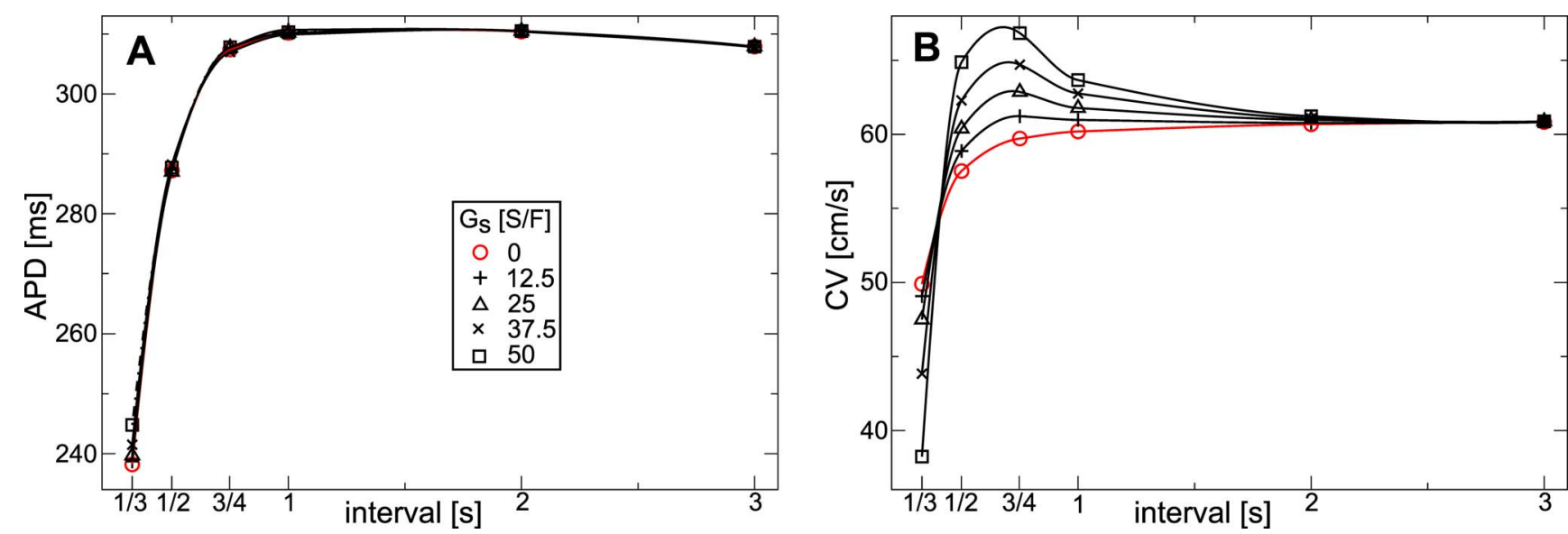

Figure 8. Dependence of restitution on stretch-activated currents in contracting medium. (A) APD restitution vs $G_{s}$. (B) CV restitution vs $G_{s}$. Same parameters were used as in Figureô 6. doi:10.1371/journal.pone.0059317.g008

the accommodation phenomenon. The linear increase of transmembrane potential shown in Figure 7 is also present in Figure 9, and we can see that it is caused by a linear increase of stretch and thus $I_{\text {sac }}$ which sets in $\approx 50 \mathrm{~ms}$ prior the upstroke. Overall, in the deforming medium the cell is affected by $I_{\text {sac }}$ only from $\approx 50 \mathrm{~ms}$ before the upstroke until $\approx 30 \mathrm{~ms}$ after the upstroke, while during constant stretch $I_{s a c}$ is always present. This explains why the effect of $G_{s}$ on the APD is much smaller in the contracting medium compared to the constantly stretched medium. Note the change in sign of $I_{\text {sac }}$ when the transmembrane potential reaches the reversal potential of the stretch activated channels $E_{s}=0 \mathrm{mV}$ (compare Eq.(12)). Thus $\boldsymbol{I}_{\text {sac }}$ has a depolarizing effect on cardiac cells prior an action potential, and can have a repolarizing effect during the action potential. From Figure 9 we can also understand the different results on the GV restitution curves for the constantly stretched and the contracting medium. In the constantly stretched medium decrease of velocity is due to the constantly depolarizing $I_{s a c}$ causing accommodation, whereas in the contracting medium, a depolarization of the medium $I_{s a c}$ sets in only about $50 \mathrm{~ms}$ prior to the upstroke (compare Figure 4), so that no significant accommodation takes place, and causes a preexcitation of the medium prior the traveling wave. As a result, for intermediate stimulation periods, an increase in $G_{s}$ causes an increase of $\mathrm{CV}$.

\section{Spiral Wave Dynamics}

We studied effects of deformation on spiral wave dynamics in our model. We initiated a spiral wave with an S1-S2 protocol in the medium, and then simulated spiral rotation for $10 s$ to avoid

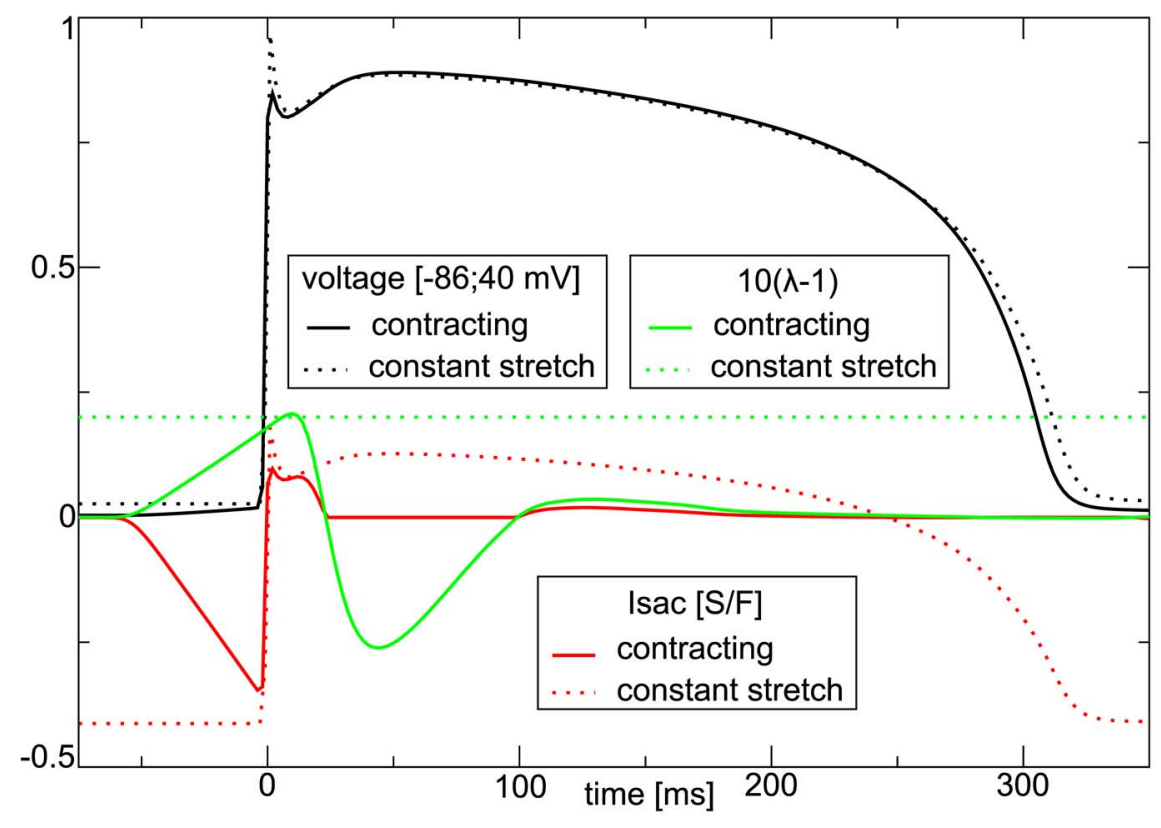

Figure 9. Effect of stretch conditions on stretch-activated currents and action potential shape. Continuous lines show variables for cell in the medium, dotted lines show variables for constantly stretched cell. The constantly stretched cell was constantly held at $\lambda=1.02$, and paced at $1 \mathrm{~Hz}$ to steady state dynamics. The other cell was in a contracting medium in the same setup as in Figureô 6, and also paced at $1 \mathrm{~Hz}$ to steady state dynamics. $G_{s}=25 \mathrm{~S} / \mathrm{F}$.

doi:10.1371/journal.pone.0059317.g009 

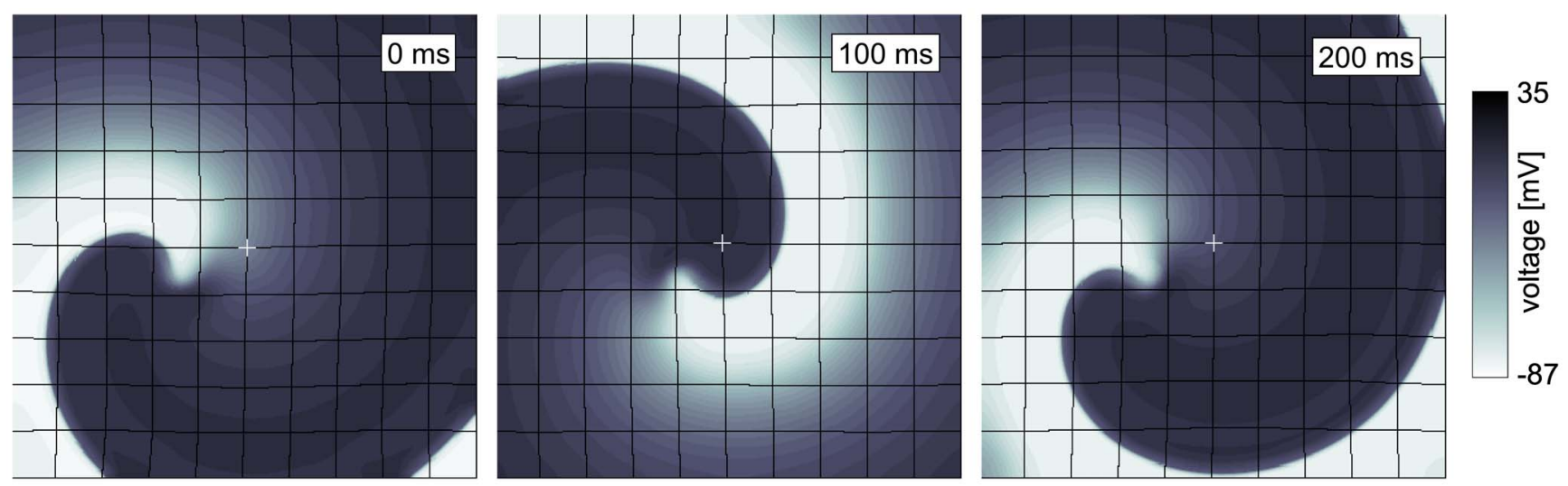

Figure 10. Illustration of spiral wave dynamics in contracting medium. Time after stretch activated current $I_{\text {sac }}$ was activated is shown top right of each subfigure. $G_{s}=10 \mathrm{~S} / \mathrm{F}$. doi:10.1371/journal.pone.0059317.g010

artifacts from the spiral initiation protocol. During this initial phase $G_{s}=0 S / F$. The values of all variables are then recorded, and used as initial conditions for the following simulations. We set $G_{s}$ to various values, in contracting and constantly stretched medium, and studied how this affects the dynamics of the rotating spiral wave. We studied spiral wave dynamics for $G_{s}$ between 0 and $20 \mathrm{~S} / \mathrm{F}$. Figure 10 illustrates the simulation experiment, it shows spiral rotation in the model for $G_{s}=10 S / F$ in the contracting medium.

We found that in the constantly stretched medium (assumed to be stretched to $\lambda_{\max }$ ) the spiral tip follows a static circular core (data not shown). Figure 11A illustrates how $I_{\text {sac }}$ affects the spiral core radius. We found that the size of the spiral wave core increases with increasing $G_{S}\left(1.04 \mathrm{~mm}\right.$ for $G_{s}=0 S / F$, and $1.17 \mathrm{~mm}$ for $\left.G_{s}=20 S / F\right)$. In Figure $11 \mathrm{~B}$ it is illustrated how the spiral period is affected by $I_{s a c}$ in the constantly stretched medium. We find, that the spiral period increases for increasing $G_{s}$. An increase of $G_{S}$ from $0 S / F$ to $20 S / F$ causes an increase of spiral period from $223 \mathrm{~ms}$ to $411 \mathrm{~ms}$. This increase in the period can be explained by elongation of APD under constant stretch conditions (Figure 9).

Figure 12A illustrates how the spiral wave rotation is affected by $I_{s a c}$ in the contracting medium. We see that in absence of $I_{s a c}$ the spiral rotates around a circular core. However, for increasing $G_{s}$ the spiral starts to drift, and drift velocity increases with an increase of $G_{s}$. All spiral tip trajectories in Figure 12A show drift for the same time interval $(\approx 4.4 s)$, and we see that the distance traveled by the spiral tip increases substantially with an increase of $G_{s}$. We use the traveled distance of the spiral tip to estimate the velocity of spiral wave drift. Figure 12B shows the velocity of spiral wave drift as a function of $G_{s}$. We see an approximately linear increase in drift velocity with increase of $G_{s}$. Figure $12 \mathrm{C}$ illustrates the effect of $I_{s a c}$ on spiral wave period. We see that increasing $G_{s}$ increases the spiral wave period: an increase of $G_{S}$ from $0 S / F$ to $20 \mathrm{~S} / \mathrm{F}$ causes an increase of spiral period from $223 \mathrm{~ms}$ to $237 \mathrm{~ms}$. This effect can be explained by the accommodation phenomenon. $I_{s a c}$ decreases the availability of sodium channels which results in a decrease of excitability which is known to increase the period of a spiral wave. However, as the effect of $I_{s a c}$ on APD is minimal (see Figure $8 \mathrm{~A}$ ) the increase of the period is also less substantial compared to the constantly stretched medium. Moreover, a decrease in excitability of a medium is known to increases the radius of a spiral core [42]. We calculated the size of the core of drifting spirals by correcting the spiral tip position data for the drift of the core, and indeed found some increase of the core radius with
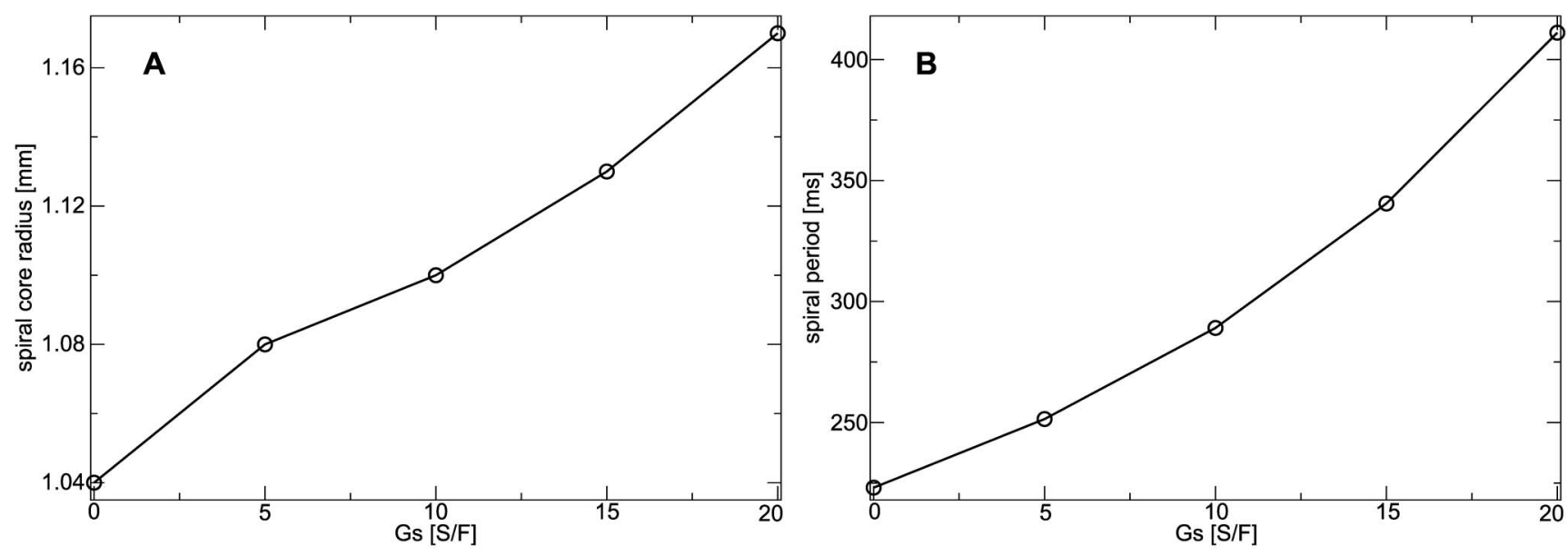

Figure 11. Dependence of spiral wave dynamics on stretch-activated currents in constantly stretched medium. (B) Spiral core radius as a function of $G_{s}$. (C) Spiral wave period as function of $G_{s}$. doi:10.1371/journal.pone.0059317.g011 


\section{A}

\section{$5 \mathrm{~mm}$}

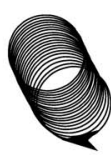

5

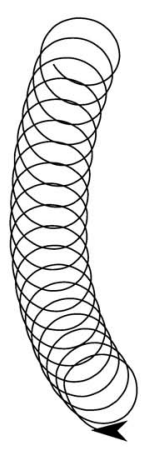

15

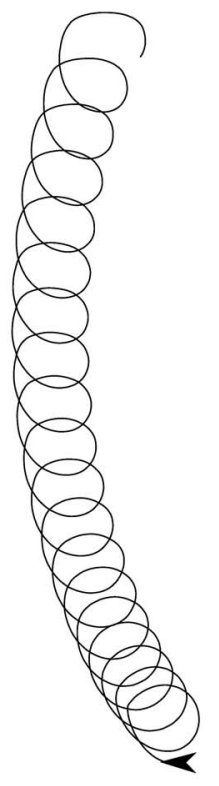

20
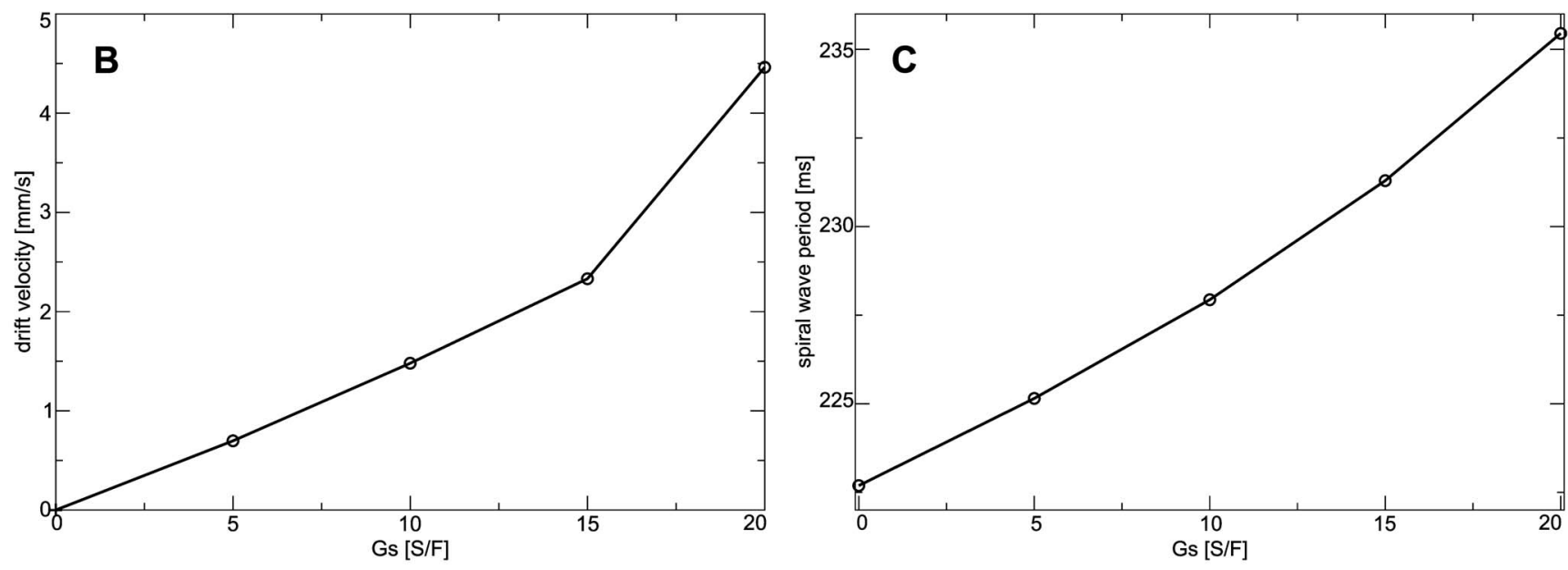

Figure 12. Dependence of spiral wave dynamics on stretch-activated currents in contracting medium. (A) Spiral tip trajectories are shown for different values of $G_{s}$. Each tip trajectory illustrates drift for $4.434 \mathrm{~s}$. Starting points and drift directions are illustrated with arrows. (B) Spiral wave drift velocity as function of $G_{s}$. Drift velocity is estimated from the tip trajectories using the distance of the spiral core position. (C) Spiral wave period as function of $G_{s}$. Spiral period was measured from the last spiral rotation.

doi:10.1371/journal.pone.0059317.g012

increasing $G_{S}\left(1.04 \mathrm{~mm}\right.$ for $G_{s}=0 S / F$, and $1.07 \mathrm{~mm}$ for $\left.G_{S}=20 S / F\right)$; however, the effect is small.

Our results on spiral wave drift in contracting tissue are qualitatively similar to that observed in [14], where a low dimensional model of cardiac tissue was applied, and the spiral drift was discussed as a so-called resonant drift mechanism [43]. Resonant-drift can be induced by a periodical variation of the medium properties such as its excitability synchronously with the spiral wave period [44]. In our model a rotating spiral wave itself periodically affects the excitability of the medium. We can understand this from Figure 10, where we can see that the fraction of the excited surface area (and thus the fraction of contracting medium) to the total surface area of the medium changes in synchrony with the spiral rotation, in turn affecting the mediums excitability properties. Therefore, we believe that in our simulations and in [14] the underlying mechanism of spiral drift is the resonant drift.

\section{Discussion}

We introduced a discrete electromechanical model of the human heart which couples a biophysical model of cardiac excitation [20,21] and tension development [22,23] with a discrete elastic mass-lattice model. We demonstrated the value of the model in an application study. We used our new model to investigate how stretch conditions and stretch-activated currents affect the heart's functioning. For this we studied how stretchactivated currents affect action potential shape, restitution properties, and spiral wave activity in a medium which we assumed constantly stretched, and a contracting medium with isometric boundary conditions. We found that stretch conditions significantly influence these properties by activating stretchactivated ion channels. In the freely deforming medium we find that the primary effects are accommodation, and preexcitation of the medium. In the constantly stretched medium we find a much 
stronger accommodation effect, no effect of preexcitation, and substantial elongation of the APD caused by depolarizing $I_{\text {sac }}$ during the recovery phase of the action potential. We found that spiral wave drift is caused in the deforming medium, whereas in the constantly stretched medium rotation dynamics is not affected, but spiral period and core size is increased.

It has been shown that the dynamics of spiral waves in the heart manifests itself in the type of cardiac arrhythmia, for example, a drifting spiral wave can induce a polymorphic ventricular tachycardia which is a known precursor for ventricular fibrillation [45]. Our results show that in addition to heterogeneity induced spiral wave drift [46-48] there is a drift due to mechano-electrical feedback which can also affect the type of cardiac arrhythmia.

Our results on restitution properties suggest that in dynamic stretch-conditions $I_{s a c}$ causes abnormal $\mathrm{CV}$ restitution due to a preexcitation in the medium. It has been shown that abnormal CV restitution can cause alternans and initiation of spiral waves $[49,50]$, and also important phenomena on spiral wave dynamics such as discordant alternans can be caused by abnormal CV restitution $[51,52]$. We expect that this mechanism of mechanically caused abnormal CV-restitution is important to understand the onset of arrhythmias due to emergent dynamic inhomogeneities.

The computation time of our mechanical model scales linearly against the number of mechanical nodes, which allows to solve the model with a higher mechanical node density and thus high spatial resolution of $I_{s a c}$ [16]. Furthermore, this computational efficiency of the discrete mechanical model allows us to update the its configuration after each electrical step $(0.02 \mathrm{~ms})$. Continuous mechanical studies on cardiac function normally solve mechanics following several electrical steps, because its more demanding numerical schemes, for example in [18] the mechanical configuration was solved following 100 electrical steps.

The passive elasticity of the heart is most commonly described by hyperelastic constitutive relations in finite element formulations of continuum mechanics, for example, the Guccione material relation in [18]. A drawback of the mass-lattice framework of the new model is its difficulty to reproduce passive mechanical properties of biological tissue with a discrete mechanical model, for example volume conservation or specific passive mechanical properties such as hyperelasticity. In contrast, these properties can be directly described in constitutive relations in continuum mechanics. The discrete electromechanical model could be extended to describe hyperelastic material relations, for example

\section{References}

1. Pool R (1990) Heart like a wheel. Science 247: 1294-1295.

2. Winfree AT (1989) Electrical instability in cardiac muscle: Phase singularities and rotors. J Theor Biol 138: 353-405.

3. Davidenko J, Pertsov A, Salomonsz R, Baxter W, Jalife J (1992) Stationary and drifting spiral waves of excitation in isolated cardiac muscle. Nature 355: 349 351.

4. Pertsov A, Davidenko J, Salomontsz R, BaxterW, Jalife J (1993) Spiral waves of excitation underlie reentrant activity in isolated cardiac muscle. Circ Res 72: 631-650.

5. Kohl P, Hunter P, Noble D (1999) Stretch-induced changes in heart rate and rhythm: Clinical observations, experiments and mathematical models. Prog Biophys Molec Biol 71: 91-138.

6. Wang Z, Taylor LK, Denney WD, Hansen DE (1994) Initiation of ventricular extrasystoles by myocardial stretch in chronically dilated and failing canine left ventricle. Circulation 90: 2022-2031.

7. Kohl P, Nesbitt A, Cooper P, Lei M (2001) Sudden cardiac death by commotio cordis: role of mechano-electric feedback. Cardiovasc Res 50: 280-289.

8. Lab M (1982) Contraction-excitation feedback in myocardium. Physiological basis and clinical relevance. Circ Res 50: 757-766.

9. Cave DM, Gazmuri RJ, Otto CW, Nadkarni VM, Cheng A, et al. (2010) Part 7: CPR techniques and devices 2010 american heart association guidelines for cardiopulmonary resuscitation and emergency cardiovascular care. Circulation 122: S720-S728. using the approach developed by Fritz et al. in [53], where a massspring model is in fact adapted to a hyperelastic material relation to describe cardiac mechanics. Moreover, also volume conservation and anisotropy of heart tissue can be introduced to discrete mechanical models $[54,55]$.

As another next step, the discrete electromechanical model can be extended to three-dimensional simulations to study the effect of mechano-electrical feedback on the dynamics of scroll waves.

Our modeling framework can potentially be used to estimate effects of mechanical or electrical components in experimental studies of wave propagation in the heart. However, it needs to be adjusted to the specific tissue type and mechanical properties of the experimental system. It can be done by changing the parameters on our model based on direct measurements.

We set up the new electromechanical model using a standard form for the stretch-activated currents $I_{s a c}$ in Eq.(12). This allows us to compare the new results to results previously achieved with electromechanical models using a continuous mechanical description, for instance results on spiral wave drift in [14]. Experimental studies showed that $I_{s a c}$ depends also on additional factors, for example, the stretch rate [56]. Our model can easily be adjusted to other formulations of $I_{s a c}$, for example, to the formulation of Jie et al. [19] which considers a stretch rate dependency. It can be interesting to compare the effect of different formulations of $I_{s a c}$ on the dynamics of wave propagation.

The effect of deformation of the medium on the metric tensor are neglected in the model, as we assume that the main resistivity between cells is constituted by gap junctions. We used this assumption also in [15], where we found that the change of the metric tensor did not affect qualitative results our study on mechanically caused pacemaking activity in a low-dimensional model.

\section{Acknowledgments}

The authors are grateful to Dr. Rikkert H. Keldermann, Dr. Martyn Nash, Dr. Kirsten ten Tusscher, and Ivan Kazbanov for valuable discussions. We thank Jan Kees van Amerongen for excellent technical support.

\section{Author Contributions}

Conceived and designed the experiments: LDW AVP. Performed the experiments: LDW. Analyzed the data: LDW AVP. Contributed reagents/ materials/analysis tools: LDW. Wrote the paper: LDW AVP.

10. Kohl P, Ravens U, editors (2003) Mechano-Electric Feedback and Cardiac Arrhythmias, Prog. Biophys. Molec. Biol., Elsevier Ltd, volume 82(1-3). 1-266.

11. Nash M, Panfilov A (2004) Electromechanical model of excitable tissue to study reentrant cardiac arrhythmias. Prog Biophys Mol Biol 85: 501-522.

12. Panfilov A, Keldermann R, Nash M (2005) Self-organized pacemakers in a coupled reactiondiffusion-mechanics system. Phys Rev Lett 95(25): 258104.

13. Keldermann RH, Nash MP, Panfilov AV (2007) Pacemakers in a reactiondiffusion mechanics system. Journal of Statistical Physics 128: 375-392.

14. Panfilov A, Keldermann R, Nash M (2007) Drift and breakup of spiral waves in reaction-diffusionmechanics systems. Proc Natl Acad Sci USA 104: 7922-7926.

15. Weise LD, Nash MP, Panfilov AV (2011) A discrete model to study reactiondiffusion-mechanics systems. PLoS ONE 6(7): e21934.

16. Weise LD, Panfilov AV (2011) New mechanism of spiral wave initiation in a reaction-diffusionmechanics system. PLoS ONE 6(11): e27264.

17. Weise LD, Panfilov AV (2012) Emergence of spiral wave activity in a mechanically heterogeneous reaction-diffusion-mechanics system. Phys Rev Lett 108: 228104.

18. Keldermann RH, Nash MP, Gelderblom H, Wang VY, Panfilov AV (2010) Electromechanical wavebreak in a model of the human left ventricle. Am J Physiol Heart Circ Physiol 299: H134-143.

19. Jie X, Gurev V, Trayanova N (2010)Mechanisms of mechanically induced spontaneous arrhythmias in acute regional ischemia. Circ Res 106: 185-192. 
20. Ten Tusscher K, Panfilov A (2006) Alternans and spiral breakup in a human ventricular tissue model. Am J Physiol Heart Circ Physiol 291: H1088-100.

21. Ten Tusscher K, Noble D, Noble P, Panfilov A (2004) A model for human ventricular tissue. Am J Physiol Heart Circ Physiol 286: H1573-H1589.

22. Niederer S, Hunter P, Smith N (2006) A quantitative analysis of cardiac myocyte relaxation: a simulation study. Biophys J 90: 1697-1722.

23. Niederer S, Smith N (2008) An improved numerical method for strong coupling of excitation and contraction models in the heart. Prog Biophys Mol Biol 96: 90111 .

24. Seth B (1935) Finite strain in elastic problems. Phil Trans R Soc Lond A 234: 231-264.

25. Verlet L (1967) Computer "experiments" on classical fluids. i. thermodynamical properties of lennard-jones molecules. Phys Rev 159: 98.

26. Mohr M (2006) A hybrid deformation model of ventricular myocardium. Karlsruhe: PhD thesis, Fridericiana University.

27. Trayanova N, Li W, Eason J, Kohl P (2004) Effect of stretch activated channels on defibrillation efficacy. Heart Rhythm 1: 67-77.

28. Vetter F, McCulloch A (2001) Mechanoelectric feedback in a model of the passively inflated left ventricle. Ann Biomed Eng 29: 414-426.

29. Hodgkin A, Huxley A (1952) A quantitative description of membrane current and its application to conduction and excitation in nerve. J Physiol 117: 500544.

30. de Tombe PP, Stienen GJM (2007) Impact of temperature on cross-bridge cycling kinetics in rat myocardium. The Journal of Physiology 584: 591-600.

31. Schargott M, Popov V, Heß M (2007) Macroscopic isotropy of two- and threedimensional elastic lattice models. Tribology International 40: 937-941.

32. Krivtsov A (1999) Constitutive equations of the nonlinear crystal lattice. $Z$ angew Math Mech 79: 419-420.

33. Mathur AB, Collinsworth AM, Reichert WM, Kraus WE, Truskey GA (2001) Endothelial, cardiac muscle and skeletal muscle exhibit different viscous and elastic properties as determined by atomic force microscopy. Journal of biomechanics 34: 1545-1553.

34. Hu H, Sachs F (1997) Stretch-activated ion channels in the heart. J Mol Cell Cardiol 29: 1511-1523.

35. Zhang Y, Youm J, Sung H, Lee S, Ryu S, et al. (2000) Stretch-activated and background nonselective cation channels in rat atrial myocytes. J Physiol 523: $607-619$.

36. Kohl P, Hunter P, Noble D (1999) Stretch-induced changes in heart rate and rhythm: clinical observations, experiments and mathematical models. Progress in Biophysics and Molecular Biology 71: 91-138.

37. Skouibine K, Trayanova N, Moore P (2000) A numerically efficient model for simulation of defibrillation in an active bidomain sheet of myocardium. Math Biosci 166: 85-100.

38. Kohl P, Day K, Noble D (1998) Cellular mechanisms of cardiac mechanoelectric feedback in a mathematical model. Can J Cardiol 14: 111-119.

39. Vahl GF, Timek T, Bonz A, Fuchs H, Dillman R, et al. (1998) Length dependence of calciumand force-transients in normal and failing human myocardium. Journal of molecular and cellular cardiology 30: 957-966.

40. Zhang Y, Sekar RB, McCulloch AD, Tung L (2008) Cell cultures as models of cardiac mechanoelectric feedback. Progress in Biophysics and Molecular Biology 97: $367-382$.
41. Franz M, Cima R, Wang D, Profitt D, Kurz R (1992) Electrophysiological effects of myocardial stretch and mechanical determinants of stretch-activated arrhythmias. Circulation 86: 968-978.

42. Panfilov AV (2009) Theory of reentry. In: Zipes DP, Jalife J, editors, Cardiac electrophysiology. From cell to bedside, 5th edition. New York: Elsevier, 329337.

43. Agladze K, Davydov V, Mikhailov A (1987) An observation of resonance of spiral waves in distributed excitable medium. JETP Lett 45: 767-769.

44. Grill S, Zykov V, Müller S (1995) Feedback-controlled dynamics of meandering spiral waves. Phys Rev Lett 75: 3368-3371.

45. Gray RA, Jalife J, Panfilov A, Baxter WT, Cabo C, et al. (1995) Nonstationary vortexlike re-entrant activity as a mechanism of polymorphic ventricular tachycardia in the isolated rabbit heart. Circulation 91: 2454-2469.

46. Ten Tusscher K, Panfilov A (2003) Reentry in heterogeneous cardiac tissue described by the Luo-Rudy ventricular action potential model. Am J Physiol Heart Circ Physiol 284: H542-H548.

47. Panfilov A, Holden A (1990) Vortices in a system of two coupled excitable fibers. Phys Lett A 147: 463-466.

48. Rudenko AN, Panfilov AV (1983) Drift and interaction of vortices in twodimensional heterogeneous active medium. Studia Biophysica 98: 183-188.

49. de Lange E, Kucera JP (2010) Alternans resonance and propagation block during supernormal conduction in cardiac tissue with decreased $[\mathrm{K}+] \mathrm{o}$. Biophysical Journal 98: 1129-1138.

50. de Lange E, Kucera JP, Qu Z (2011) Supernormal excitability causes alternans, block, wavebreak and reentry in cardiac tissue. Biophysical Journal 100: 435a.

51. Echebarria B, Rder G, Engel H, Davidsen J, Br M (2011) Supernormal conduction in cardiac tissue promotes concordant alternans and action potential bunching. Physical Review E 83: 040902.

52. Kwon O, Kim TY, Lee KJ (2010) Period-2 spiral waves supported by nonmonotonic wave dispersion. Physical review E, Statistical, nonlinear, and soft matter physics 82: 046213.

53. Fritz T, Jarrousse O, Dössel O (2009) Adapting a mass-spring system to energy density function describing myocardial mechanics. In: Vander Sloten J, Verdonck P, Nyssen M, J H, editors, 4th European Conference of the International Federation for Medical and Biological Engineering. Berlin, Heidelberg: Springer, 2003-2006.

54. Bourguignon D, Cani MP (2000) Controlling anisotropy in mass-spring systems. In: Eurographics Workshop on Computer Animation and Simulation (EGCAS). Springer-Verlag, Springer Computer Science, 113-123. URL http://wwwevasion.imag.fr/Publications/2000/BC00. Proceedings of the 11th Eurographics Workshop, Interlaken, Switzerland, August 21-22, 2000.

55. Mohr M, Seemann G, Sachse F, Dössel O (2003) Modeling of myocardial deformation with an extended spring mass system. Biomedical Engineering 48(1): $6-7$.

56. Nishimura S, Kawai Y, Nakajima T, Hosoya Y, Fujita H, et al. (2006) Membrane potential of rat ventricular myocytes responds to axial stretch in phase, amplitude and speed-dependent manners. Cardiovascular research 72: 403-411. 